\title{
Article \\ Thickness Measurement for Glass Slides Based on Chromatic Confocal Microscopy with Inclined Illumination
}

\author{
Qing Yu* , Yali Zhang, Wenjian Shang, Shengchao Dong, Chong Wang, Yin Wang, Ting Liu and Fang Cheng \\ College of Mechanical Engineering and Automation, Huaqiao University, Xiamen 361021, China; \\ 19013080066@stu.hqu.edu.cn (Y.Z.); 19013080059@stu.hqu.edu.cn (W.S.); 20013080011@stu.hqu.edu.cn (S.D.); \\ 20013080044@stu.hqu.edu.cn (C.W.); yin.wangyin@hqu.edu.cn (Y.W.); liut14@hqu.edu.cn (T.L.); \\ chengfang@hqu.edu.cn (F.C.) \\ * Correspondence: yuqing@hqu.edu.cn
}

Citation: Yu, Q.; Zhang, Y.; Shang, W.; Dong, S.; Wang, C.; Wang, Y.; Liu, T.; Cheng, F. Thickness Measurement for Glass Slides Based on Chromatic Confocal Microscopy with Inclined Illumination. Photonics 2021, 8, 170. https://doi.org/10.3390/ photonics 8050170

Received: 18 April 2021

Accepted: 18 May 2021

Published: 20 May 2021

Publisher's Note: MDPI stays neutral with regard to jurisdictional claims in published maps and institutional affiliations.

Copyright: (c) 2021 by the authors. Licensee MDPI, Basel, Switzerland. This article is an open access article distributed under the terms and conditions of the Creative Commons Attribution (CC BY) license (https:// creativecommons.org/licenses/by/ $4.0 /)$.

\begin{abstract}
Chromatic confocal microscopy is a widely used method to measure the thickness of transparent specimens. In conventional configurations, both the illumination and imaging axes are perpendicular to the test specimen. The reflection will be very weak when measuring hightransparency specimens. In order to overcome this limitation, a special chromatic confocal measuring system was developed based on inclined illumination. This design was able to significantly improve the signal-to-noise ratio. Compared with conventional designs, the proposed system was also featured by its biaxial optical scheme, instead of a coaxial one. This biaxial design improved the flexibility of the system and also increased the energy efficiency by avoiding light beam splitting. Based on this design, a prototype was built by the authors' team. In this paper, the theoretical model of this specially designed chromatic confocal system is analyzed, and the calculating formula for the thickness of transparent specimen is provided accordingly. In order to verify its measurement performance, two experimental methodology and results are presented. The experimental results show that the repeatability is better than $0.54 \mu \mathrm{m}$, and the axial measurement accuracy of the system could reach the micron level.
\end{abstract}

Keywords: chromatic confocal measurement; thickness measurement; inclined illumination; color camera

\section{Introduction}

Chromatic confocal measurement technology is an optical approach, which is widely used in surface 3D reconstruction [1,2], defect detection [3,4], roughness quantification [5,6], displacement sensing [7,8], and thickness measurement [9]. Chromatic confocal measurement technology evolved from traditional laser confocal measurement technology [10]. Instead of physically scanning along the optical axis and detecting the focal point, the chromatic confocal measurement system measures the surface topography using a series of dispersed light components with different wavelengths. It shortens the scan time, so it has the advantages of fast measurement, high precision, and high resolution. In recent years, many efforts have been made to improve the performance of the chromatic confocal measurement.

For efficiency improvement, efforts have been focused on lateral scanning mechanisms. In 2015, Hillenbrand $M$ et al. [11] presented a chromatic confocal matrix sensor with actuated pinhole arrays for 3D object snapshot acquisition, which significantly improved the lateral resolution by applying pinhole arrays. In 2020, Luo D et al. [12] proposed a direct area scanning method for 3D surface profilometry based on a tilted focal field. The tilting angle was specifically chosen according to the numerical aperture of the system. The method was more than 300 times faster than the conventional pinhole scanning mechanism, while the axial uncertainty increased by a factor of 2.5. In 2020, Li S B et al. [13] used DMD (Digital Micromirror Device) as an alternative to pinhole array in order to obtain areal measurement by horizontally switching the mirror units instead of mechanical scanning. 
For accuracy improvement, the presented works focused on optical model optimization. Hillenbrand $\mathrm{M}$ et al. [14] presented a novel mathematical function, called the intensity point-spread function, to reshape the characteristic curve of the chromatic confocal system. Using this method, the measurement repeatability and computation efficiency could be improved. In 2019, Lu et al. [15] proposed a hybrid radial basis function network method to characterize the displacement response for chromatic confocal microscopy, so as to improve the measurement accuracy. In 2021, Bai J et al. [16] proposed a self-reference dispersion correction to correct the dispersion of the unstable light source or the alterable specimen surface. The results demonstrated that the correction significantly improved the robustness and accuracy against different light sources and specimen surfaces in chromatic confocal displacement measurement.

In recent years, chromatic confocal technology has also been applied to measure the thickness of transparent specimens. In 2010, Antonin M et al. [17] dealt with the induced measurement errors when measuring the thickness of a plane-parallel plate and the central thickness of a lens. They derived a quantitative evaluation of these errors and a method was presented for minimizing the influence of these errors. In 2017, Boettcher T et al. [18] proposed a new hybrid single-shot imaging scheme that combined chromatic confocal and interference technology, to simultaneously measure the thickness and refractive index for translucent materials. In 2018, Zhang K et al. [19] established a thickness measurement model by adding an auxiliary reflector below the specimen. This model was able to significantly enlarge the tolerance of the specimen placement. The experimental comparison showed that the proposed method was able to achieve a measurement accuracy of $0.25 \mu \mathrm{m}$. In 2020, Jia F et al. [20] proposed an adaptive modal decomposition method to separate multiple peaks when they had overlapping areas. This method showed the advantage of accuracy, especially when the multiple peaks had overlapping areas.

In those systems discussed above, the illuminating path and the imaging path formed a coaxial structure. In order to meet the requirements of different applications, inclined measurement structures have been proposed in recent years. In 2012, Taphanel et al. [21] proposed a new measurement principle for this purpose, combining the chromatic confocal technique and the triangulation principle. They constructed an optical line scan measurement device to measure the 3D topology of specular and diffuse surfaces in absolute coordinates. In 2015, Taphanel et al. [22] proposed a multispectral system by applying a monochrome camera in combination with a multiplex light source. The scan speed could be as high as the frame rate, which enabled high-speed measurements. In 2020, Seung et al. [23] proposed spectroscopic imaging ellipsometry that was able to measure spectral ellipsometric signals in the entire field of view simultaneously without areal scanning or the operation of polarization devices. By comparing the thickness results of the $\mathrm{SiO}_{2} / \mathrm{Si}$ specimen measured using a commercial ellipsometer, the proposed method was verified. However, the measurement range of the ellipsometry technique was too small to adapt to the measurement requirements for macro objects.

Compared with the inclined structural light path mentioned above, for transparent specimen measurement, the conventional light path showed two main disadvantages. Firstly, the imaging axis was perpendicular to the measured surface. For a high transparency and low-refraction material, the reflected light will be very weak. The low signal-tonoise ratio will significantly affect the measurement performance. Secondly, the efficiency of the light energy is quite low. The application of a beam splitter makes the receiver have only $1 / 4$ of the energy of the light source. To solve these limitations, a novel chromatic confocal system with inclined illumination to measure the thickness of transparent specimen was developed by the authors' team and will be presented in this paper. 


\section{System Principle and Optical Path Design}

\subsection{Proposal of Chromatic Confocal Measurement with Inclined Illumination}

\subsubsection{Principle of Chromatic Confocal Measurement}

As a non-contact optical measurement method, chromatic confocal technology can obtain the surface topographic image of the specimen without mechanical axial scanning. The schematic diagram of chromatic confocal technology is shown in Figure 1. The polychromatic light beam emitted from the white light source passes through an illumination pinhole (pinhole 1 in Figure 1), which modulates the light beam into a point light. Then, the light beam with different wavelengths is distributed along the optical axis by the dispersion tube lens. The dispersed lights are then focused at the different axial positions by the objective. Being reflected by the specimen surface, only the light beam from the focal position can pass through the detection pinhole (pinhole 2 in Figure 1) and arrive at the detector with a peak intensity. By detecting the wavelength with a peak intensity, the height of the current measurement point can be determined.

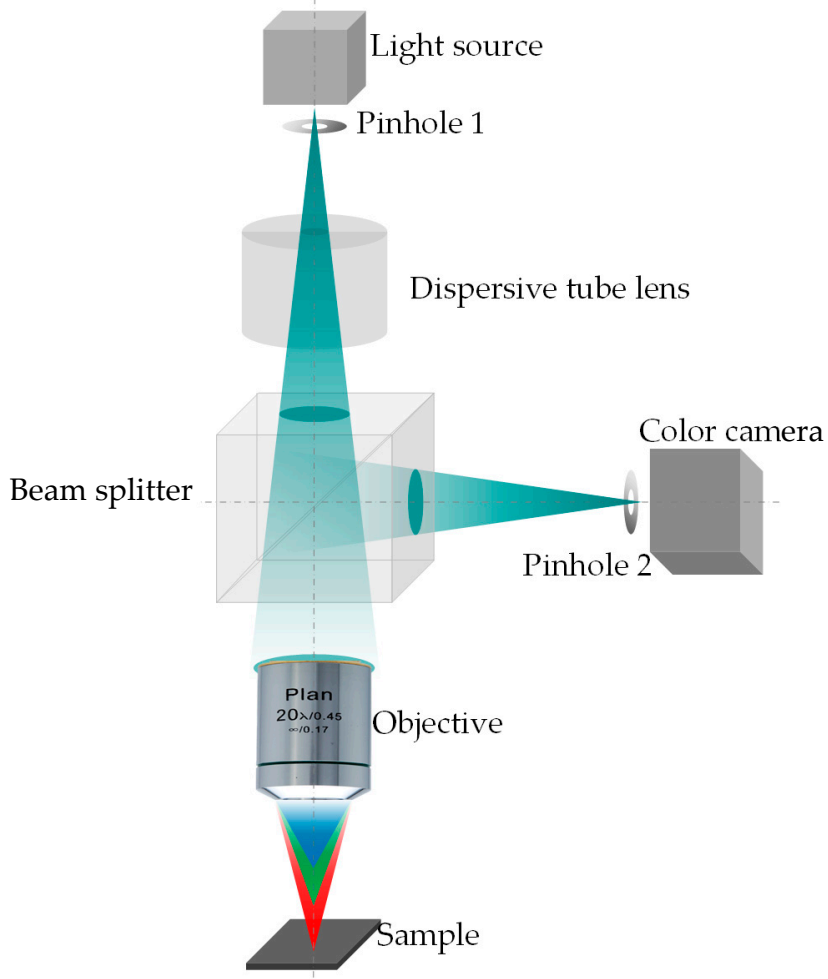

Figure 1. Principle of the chromatic confocal measurement method.

\subsubsection{Principle of Chromatic Confocal Measurement with Inclined Illumination}

In a conventional chromatic confocal measuring system, the optical axis is set to be perpendicular to the specimen surface, as mentioned in Section 2.1.1. When measuring a transparent specimen, it can capture the upper and lower surfaces by detecting two peaks of intensity. For thin sheet measurement, these two peaks may overlap to some extent, as shown in Figure 2. One of the solutions is to reduce the size of pinhole 2 to improve the detection sensitivity. However, the tradeoff is the weaker imaging light and higher alignment difficulty.

The other solution is to separate the imaging path from the illumination one, as shown in Figure 3. 


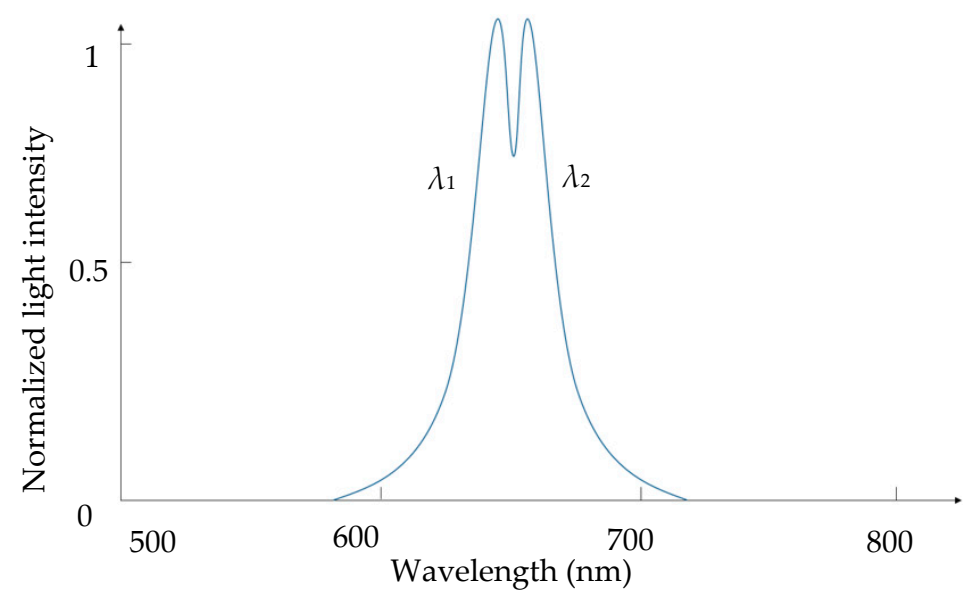

Figure 2. Overlap of the signals from the top and bottom surfaces of transparent specimen $\left(\lambda_{1}\right.$ and $\lambda_{2}$ represent two different wavelengths, respectively).

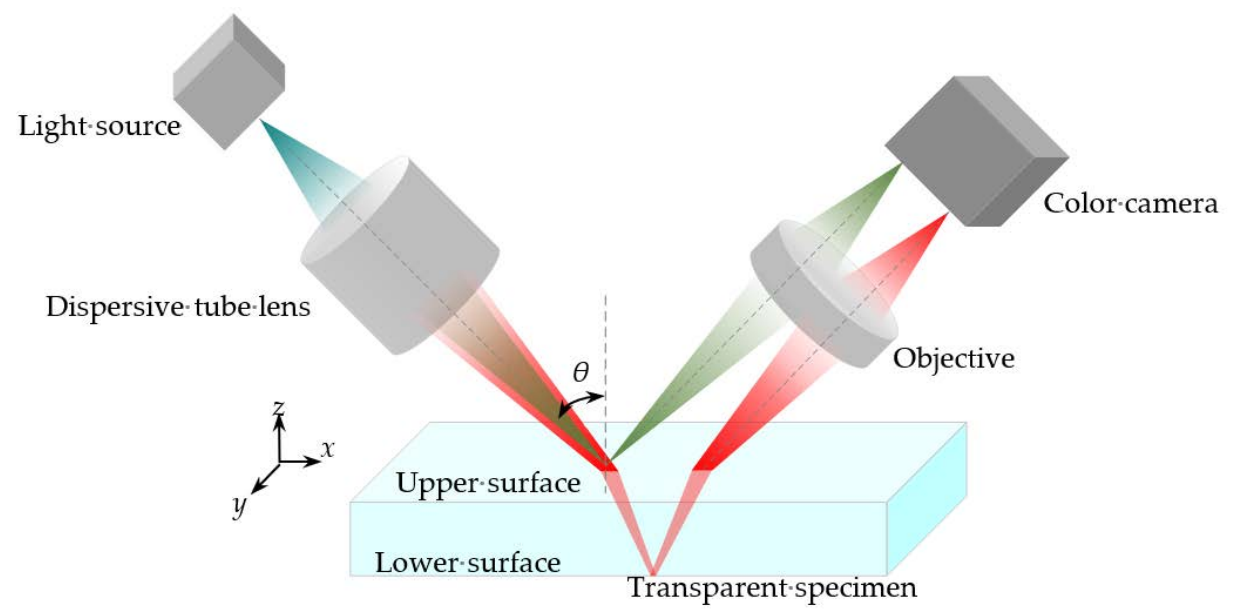

Figure 3. Separation of these two signals from the upper and lower surfaces of a transparent specimen ( $\theta$ is the inclined angle between the optical axis of incident light and the normal direction of the specimen surface).

In this design, the optical axis of the illumination path is not perpendicular to the specimen surface, as it is set in a conventional confocal system. Under this situation, the light beam from the light source, which is HL-2000-FHSA of the Ocean Optics, passes through a dispersive tube lens and reaches the upper surface. Then, the reflected light beams from the upper and lower surfaces of the specimen are focused at the different locations of the detector, and these two optical paths are parallel, instead of coaxial, to each other. In order to keep the confocal structure, a new conjugated relationship should be built for the illumination pinhole, the imaging focal point, and the detection pinhole. Because the imaging light is no longer coaxial, the method of point detection in the conventional chromatic confocal measurement system is not applicable in the proposed system. An inclined detection method is needed.

\subsection{Theory of Chromatic Confocal Measurement with Inclined Illumination}

2.2.1. Theoretical Analysis

According to the chromatic confocal microscope theory, a prototype with an inclined illumination was built, and the upper surface of the transparent specimen was set as the test specimen, as shown in Figure 3. 
In the conventional chromatic confocal measurement technology, the normalized light intensity at the confocal pinhole can be expressed as follows:

$$
I(u)=\left[\frac{\pi a^{2}}{\lambda f} \operatorname{sinc}\left(\frac{u^{4}}{4}\right)\right]^{4}
$$

where $u$ is the normalized axial coordinate, $\lambda$ is the wavelength of the incident light, $a$ is the exit pupil radius of the imaging lens, and $f$ represents the focal length of the lens. The normalized axial coordinate $u$ can be expressed as follows:

$$
u=\frac{2 \pi}{\lambda} \Delta z \frac{a^{2}}{f^{2}}
$$

In Equation (2), $\Delta z$ is the defocusing amount, which is determined by the response function of axial light intensity in a chromatic confocal measurement system.

Equation (1) indicates that every light spot with a different wavelength corresponds to an axial light intensity response, which is largest at the position of the focused point, and decreases with the increasing of the defocusing amount, as shown in Figure 4.

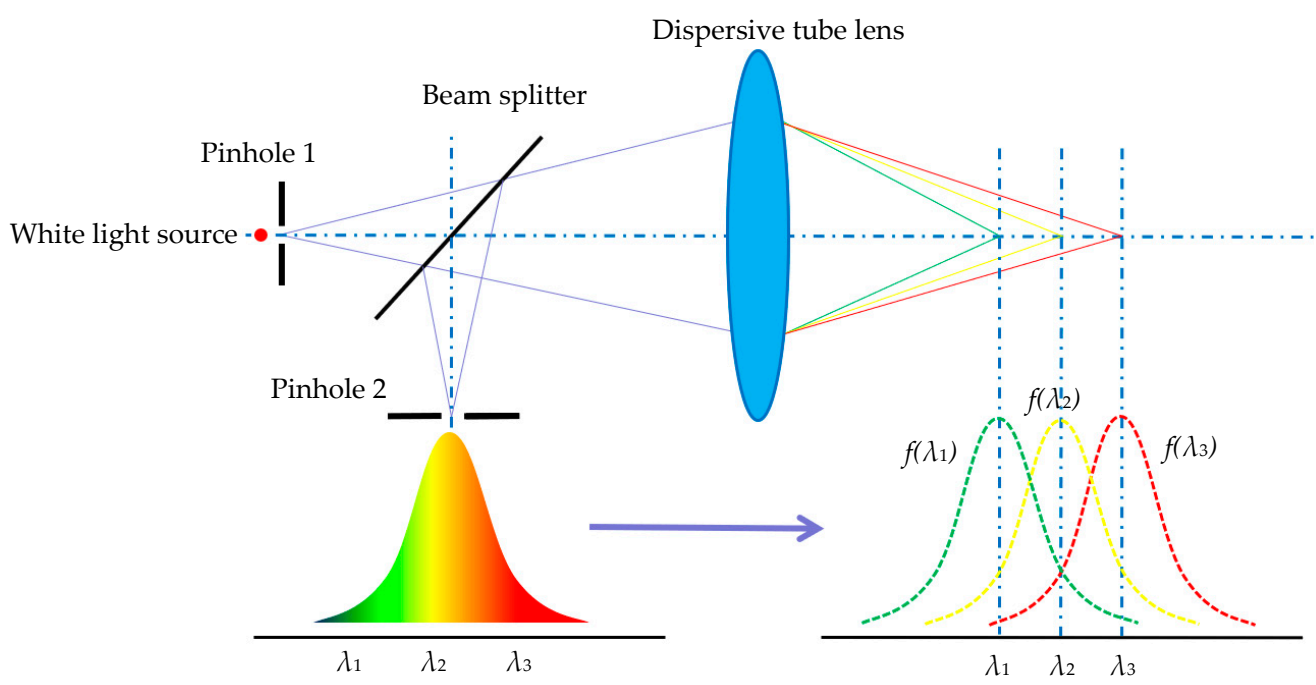

Figure 4. Schematic diagram of the intensity response.

In an ideal chromatic confocal system, the axial positions are linearly related to the wavelengths of the focused lights:

$$
f\left(\lambda_{i}\right)=h_{0}+k \lambda_{i}
$$

where $f\left(\lambda_{i}\right)$ represents the optical axial position, $\lambda_{i}$ represents the wavelength of the focused light, and $h_{0}$ and $k$ are constants. For a beam of light with a wavelength $\lambda_{i}$, the defocusing amount is as follows:

$$
\Delta h=f\left(\lambda_{i}\right)-f\left(\lambda_{0}\right)=k\left(\lambda_{i}-\lambda_{0}\right)
$$

In Figure 3, the inclined angle between the optical axis of illumination path and the normal direction of the specimen surface is defined as $\theta$. Thus, the relationship between the displacement of the focused point $\Delta h$ and that of the specimen $\Delta z\left(\lambda_{i}\right)$ can be expressed as follows:

$$
\Delta z\left(\lambda_{i}\right)=\Delta h \cdot \cos \theta
$$


Substituting Equations (3)-(5) into Equation (1), the light intensity response function of any wavelength at the focused point is shown as follows:

$$
I\left(\lambda_{i}\right)=\left[\frac{\pi a^{2}}{\lambda_{i}\left(h_{0}+k \lambda_{i}\right)} \operatorname{sinc}\left(\frac{\pi a^{2} k\left(\lambda_{i}-\lambda_{0}\right) \cos \theta}{2 \lambda_{i}\left(h_{0}+k \lambda_{i}\right)^{2}}\right)\right]^{4}
$$

The light intensity distribution of Equation (6) is simulated, which is shown in the black curve in Figure 5. The curve consists of all the peak points of light intensity at every wavelength from $400-700 \mathrm{~nm}$, and each curve at every wavelength can be calculated and plotted with the conventional laser confocal formula, as shown in Equation (7). To understand the axial intensity distribution of the chromatic confocal system easily, several groups of light intensity curves at wavelengths of $450 \mathrm{~nm}, 500 \mathrm{~nm}, 550 \mathrm{~nm}, 600 \mathrm{~nm}$, and $650 \mathrm{~nm}$ were simulated, as shown in the curves with different colors in Figure 5.

$$
I(z)=\left[\frac{\sin \left(\frac{u^{\prime}}{4}\right)}{\frac{u^{\prime}}{4}}\right]^{4}
$$

where $u^{\prime}$ is the normalized axial optical coordinate, which can be expressed as follows:

$$
u^{\prime}=\frac{2 \pi}{\lambda} z \frac{a^{2}}{f^{2}}
$$

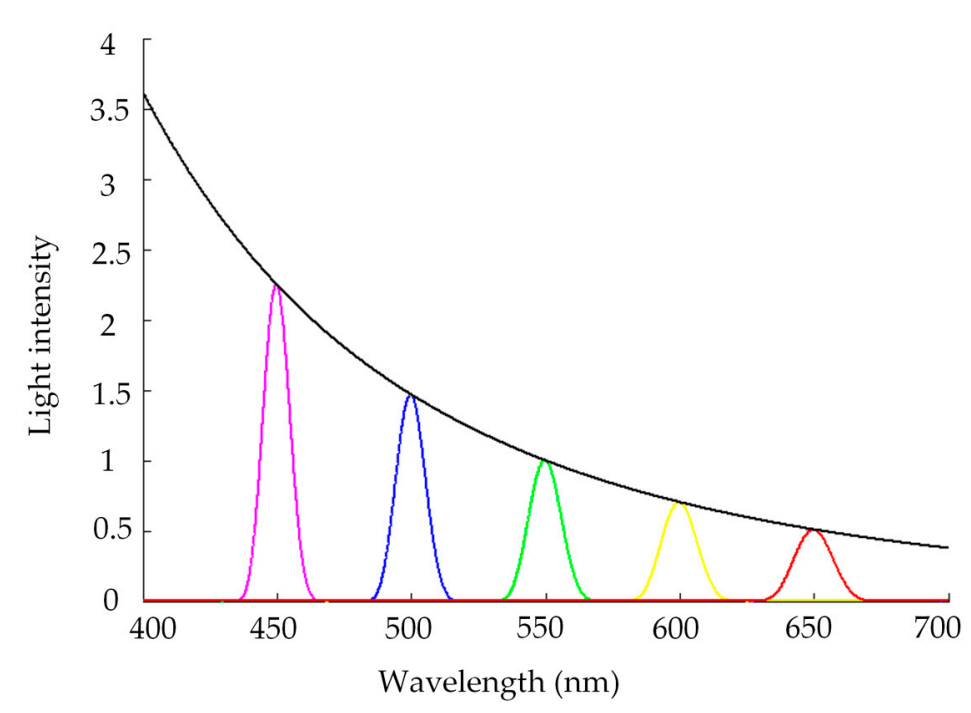

Figure 5. Light intensity distribution with different wavelengths.

As mentioned above, the wavelength detected with the peak intensity is related to the axial position of the light spot in the chromatic confocal system, as a result, the chromatic confocal system with inclined illumination also has good spectral frequency selection characteristics.

2.2.2. Thickness Calculation Model in the Chromatic Confocal System with Inclined Illumination

In Section 2.1.2, the theoretical model of the proposed system was analyzed. In this section, the thickness calculation model for a transparent specimen is discussed.

As shown in Figure 6, there are two wavelength components $\left(\lambda_{0}\right.$ and $\left.\lambda_{i}\right)$ focused on the upper and lower surfaces, with the focal points of $O$ and $B$, respectively. If there was no test specimen, the light beam with wavelength $\lambda_{i}$ would be focused at point $C$. 


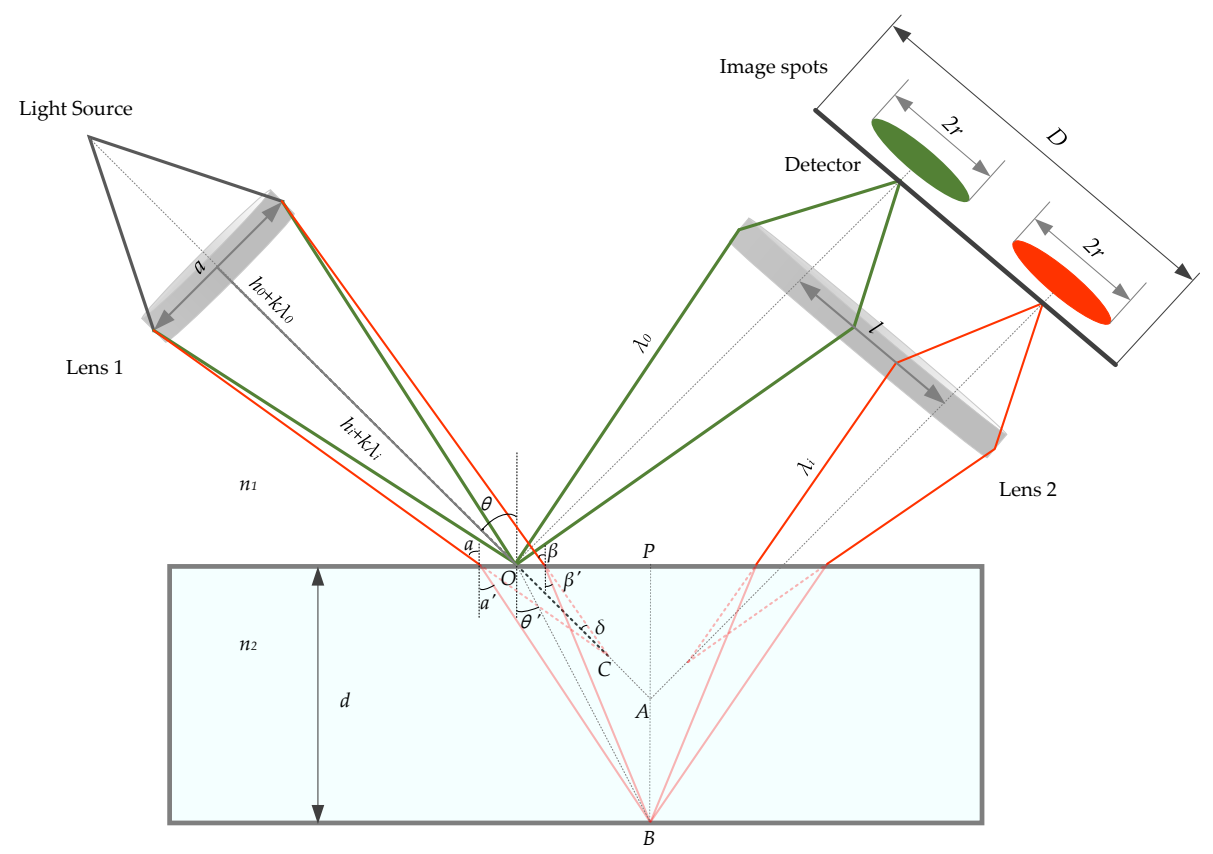

Figure 6. Thickness calculation model of a transparent specimen. The letters in the picture represent the following meanings: $\boldsymbol{n}_{\mathbf{1}}$ : the refractive index of the air; $\boldsymbol{n}_{\mathbf{2}}$ : the refractive index of the transparent specimen; $\lambda_{0}$ : the wavelength of green light; $\lambda_{i}$ : the wavelength of red light; $h_{0}+k \lambda_{0}$ : the distance between point $\mathrm{O}$ and the lens $1 ; h_{0}+k \lambda_{i}$ : the distance between point $\mathrm{C}$ and the lens $1 ; \boldsymbol{a}$ : the diameter of lens 1 ; $d$ : the thickness of the transparent specimen; $D$ : the imaging size of detector imaging surface; $r$ : the radius of the image spots; $l$ : the center distance of the imaging spots; $\theta$ : the inclined angle of the system; $\boldsymbol{\theta}^{\prime}$ : the inclined angle of the system after refraction; $\delta$ : the optical axis of incident light and light beam with wavelength $\lambda_{i} ; \alpha$ : the incidence angle of the left light beam with wavelength $\lambda_{i}$; $\alpha^{\prime}$ : the refraction angle of the left light beam with wavelength $\lambda_{i} ; \beta$ : the incidence angle of the right light beam with wavelength $\lambda_{i} ; \beta^{\prime}$ : the refraction angle of the right light beam with wavelength $\lambda_{i}$.

The thickness of the transparent specimen $d$ can be expressed as follows:

$$
d=O A \cdot \cos \theta \cdot \frac{(\tan \alpha-\tan \beta)}{\left(\tan \alpha^{\prime}-\tan \beta^{\prime}\right)}
$$

Substituting Equation (4) into Equation (9),

$$
d=k\left(\lambda_{i}-\lambda_{0}\right) \cdot \cos \theta \cdot \frac{(\tan \alpha-\tan \beta)}{\left(\tan \alpha^{\prime}-\tan \beta^{\prime}\right)}
$$

$\alpha$ and $\beta$ can be calculated by $\theta$ and $\delta$ :

$$
\left\{\begin{array}{l}
\alpha=\theta+\delta \\
\beta=\theta-\delta
\end{array}\right.
$$

$\delta$ is related to the diameter of the dispersion tube lens and the distance between point $C$ and the dispersion tube lens. In the author's system, these two values are $a$ and $h_{0}$, respectively. Thus, $\delta$ can be expressed by the following:

$$
\delta=\arctan \left[\frac{a}{2\left(h_{0}+k \lambda_{i}\right)}\right]
$$

Finally, in order to make the expression more concise, Equation (10) is preserved. However, as in the analysis mentioned above, for the actual calculation of thickness, Equations (11) and (12) are both necessary. 


\subsubsection{Analysis for the Influence of $\theta$ on Measurement Range of Thickness}

In this proposed chromatic confocal model, the inclined angle $\theta$ is critical. Therefore, the relationship between the thickness $d$ and the angle $\theta$ is worth discussion. As shown in Figure 6, there is a geometric relationship between the thickness $d$ and the angle $\theta$, which can be expressed as follows:

$$
d=P B=\frac{O P}{\sin \theta^{\prime}}=\frac{n_{1} \cdot l \cdot \sin \theta}{\tan \left(\arcsin \left(\frac{\sin \theta}{n_{2}}\right)\right)}
$$

In our system, $n_{1}$ is 1 . Based on Equation (13), the curves of $d$ can be plotted as Figure 7.

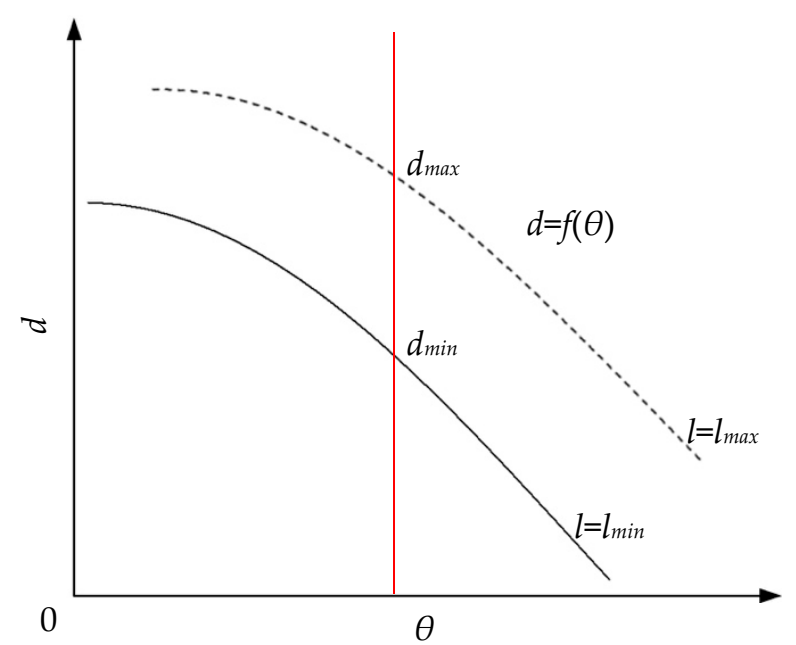

Figure 7. The relationship between the thickness $d$ and the angle $\theta$.

There are two curves in Figure 7. The curve with a solid line and dotted line represent the minimum and maximum thickness that the system can measure, respectively. Obviously, both curves will decrease as the angle $\theta$ increasing. Now, if the angle $\theta$ is fixed, as shown with the red line in Figure 7 , we will get $d_{\max }$ and $d_{\min }$. As indicated by Equation (13), $d_{\max }$ and $d_{\min }$ are both decided by $l$. Therefore, it is necessary to discuss the value range of $l$. There are two light spots with red and green in Figure 6, which are reflected by the upper and lower surfaces, respectively. When these two light spots are circumscribed, they can be distinguished, and $l$ has the minimum value, as follows:

$$
l_{\min }=2 r
$$

$r$ represents the radius of the light spot.

As the thickness of the transparent specimen increases, the distance between the light spots received by the detector will also increase. However, the size of the detector is limited, so when these two light spots arrive at the edge of the detector seperately, $l$ has the maximum value:

$$
l_{\max }=D-2 r
$$

$D$ represents the imaging size of the detector.

Considering the specific parameters of the experimental device, such as the linear range of the dispersive tube lens, the maximum and minimum values of $l$ can be obtained. The dispersive tube lens is developed in house, and its linear range is known as $1100 \mu \mathrm{m}$, which is smaller than $D-2 r$. Combining those quantities in Section 3.1.1, the values of $d_{\min }$ and $d_{\max }$ can be calculated. 


\subsubsection{Color Conversion Algorithm}

The images collected by the color camera contain $R, G$, and $B$ values, which make the images show different colors. As discussed in the above sections, the axial displacement of the specimen is related to the wavelength detected with the peak intensity. However, the wavelength value cannot be directly obtained through the RGB image. Thus, a conversion algorithm is needed to translate the color information into wavelength values. The wavelength information can be obtained by converting the RGB space into the HSI space, in which the $H$ value is directly related to the wavelength. This conversion is shown in Figure 8:

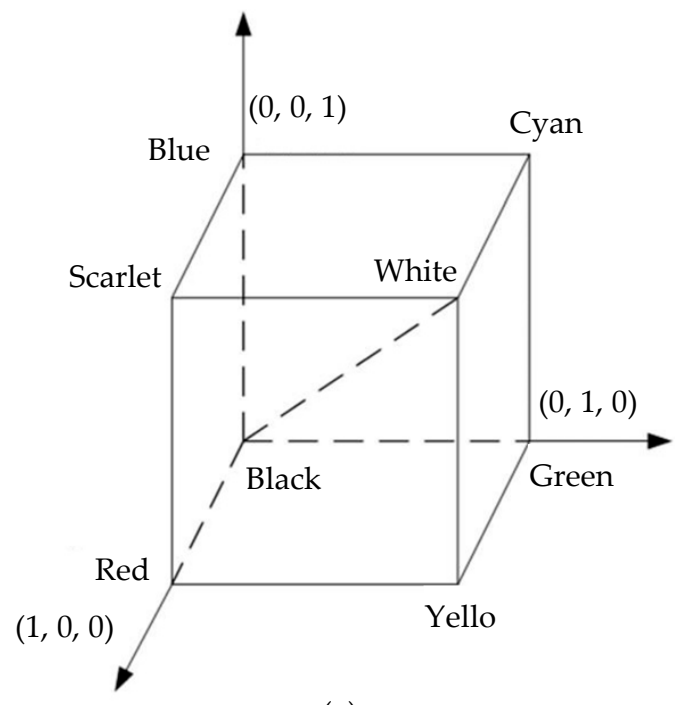

(a)

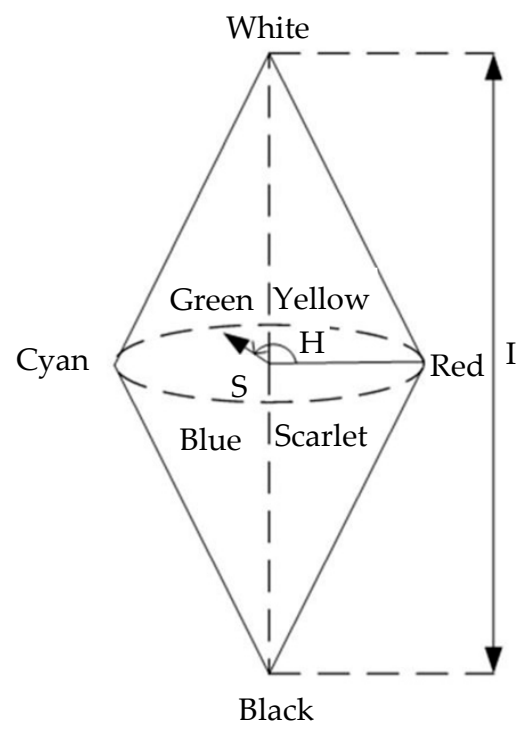

(b)

Figure 8. Principle of color conversion algorithm. (a) RGB space; (b) HSI space.

In the HSI space, $H$ can be expressed as follows:

$$
H=\left\{\begin{array}{cc}
\theta, & G \geq B \\
2 \pi-\theta, & G<B
\end{array}\right.
$$

where $\theta=\cos ^{-1}\left(\frac{(R-G)+(R-B)}{2 \sqrt{(R-G)^{2}+(R-B)(G-B)}}\right)$

Using Equation (16), the $R, G$, and $B$ information obtained by the color camera can be converted to $H$ value. Therefore, Equation (3) will become the following:

$$
f\left(H_{i}\right)=h_{1}+k_{1} H_{i}
$$

The thickness of the transparent specimen $d$ becomes the following:

$$
d=k_{1}\left(H_{i}-H_{0}\right) \cdot \cos \theta \cdot \frac{(\tan \alpha-\tan \beta)}{\left(\tan \alpha^{\prime}-\tan \beta^{\prime}\right)}
$$

\section{Experimental Analysis}

\subsection{Setup and Calibration Experiment of the System}

\subsubsection{The Selection of Angle $\theta$}

As mentioned in Section 2.2.3, $l_{\min }$ and $l_{\max }$ can be calculated through the size of light spot, the size of detector and the linear range of the dispersive tube lens. In the experiments, thicknesses of specimens were about $180 \mu \mathrm{m}$ and $1000 \mu \mathrm{m}$, respectively; so the $d_{\min }$ and $d_{\max }$ were set as 50 and $1100 \mu \mathrm{m}$, respectively. Therefore, the angle $\theta$ can be calculated as $39^{\circ}$ by Equation (13). 


\subsubsection{Setup of the System}

Based on these theory analyses mentioned above, a chromatic confocal measurement device with inclined illumination was built, as shown in Figure 9. In this measurement device, an in-house developed dispersion tube lens was used to produce axial dispersion, and two objectives were placed before the specimen. The parameters of the two objectives are shown in Table 1. The motor diver is the T-Cube DC Servo Controller (TDC001), which is used to push the transparent specimen in the direction of the yellow arrow shown in Figure 9. The computer is used to control the movement of the motor and is also used to connect the camera to get the RGB image. In the detection part, a color camera is applied as the receiver, whose model is Balser acA2440-35uc.

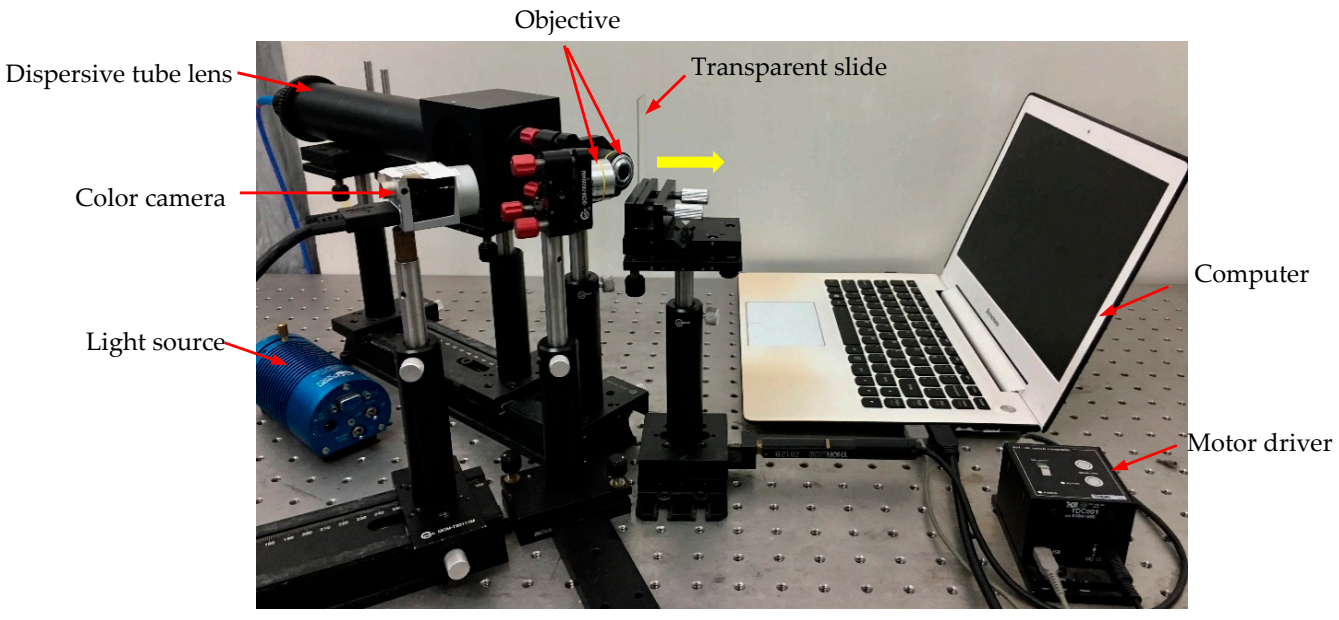

Figure 9. Diagram of the chromatic confocal system with inclined illumination.

Table 1. The parameters of the objectives.

\begin{tabular}{cc}
\hline Objective Parameters & Value \\
\hline magnification & $10 \times$ \\
N.A. value & 0.2 \\
\hline
\end{tabular}

\subsubsection{Calibration Experiment of the System}

To establish the relationship between the position of the specimen surface and the information of the image color, a calibration experiment was performed in this system. A slide was used as the specimen in the experiment. The data of 16 positions along the yellow arrow were recorded, as shown in Figure 10. The images were captured by the color camera and converted to $H$ values by the in-house developed color conversion algorithm in Section 2.2.4.

In Figure 9, as the measured transparent specimen gradually moves away from the objective, the color received by the color camera will gradually change from green to red. As shown in Figure 10, 16 groups of pictures were captured in the calibration experiment that could indicate the color change from the upper and lower surfaces of the transparent glass slide in the whole calibration process. In the author's system, a circle with a diameter of 100 pixels was selected as the "pinhole". Thus, the average $H$ value of the circle in the center of one picture was calculated to characterize the whole light spot. Figure 10 shows the pixel-processed calibration image, which is a circle with a diameter of 100 pixels. "The lower" in Figure 10 represents the color change reflected from the lower surface of the transparent specimen, and "the upper" represents the color change reflected from the upper surface of the transparent specimen. In the calibration experiment, only the images from the upper surface of the slide were recorded and processed.

The average $H$ values of these 16 light spots in Figure 10 were calculated, and the axial displacement was measured by the inductance micrometer Tesa TT80. The calibration 
curve before fitting is shown in Figure 11a. Within $600-1700 \mu \mathrm{m}, 12$ points were selected for fitting. The experimental data are presented in Table 2 and the linear fitting result is plotted in Figure 11b.

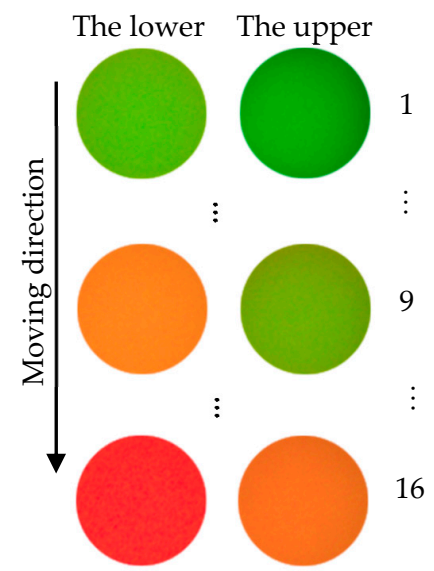

Figure 10. The color change of the calibration experiment.

Table 2. Calibration result calculated using the data from the upper surface of the slide.

\begin{tabular}{cc}
\hline Axial Displacement $(\mu \mathrm{m})$ & $\boldsymbol{H}$ Value \\
\hline 1700.00 & 22.41 \\
1600.00 & 28.26 \\
1500.00 & 34.78 \\
1400.00 & 42.24 \\
1300.00 & 50.11 \\
1200.00 & 58.11 \\
1100.00 & 66.66 \\
1000.00 & 74.32 \\
900.00 & 82.11 \\
800.00 & 91.04 \\
700.00 & 102.65 \\
600.00 & 113.16 \\
500.00 & 119.32 \\
400.00 & 123.91 \\
\hline
\end{tabular}

In the linear fitting, the standard square value of the fitting curve was 0.99 , and the relationship between the axial displacement of the slide $f(H)$ and the $H$ value is as below:

$$
f(H)=1924.90-12.14 H
$$




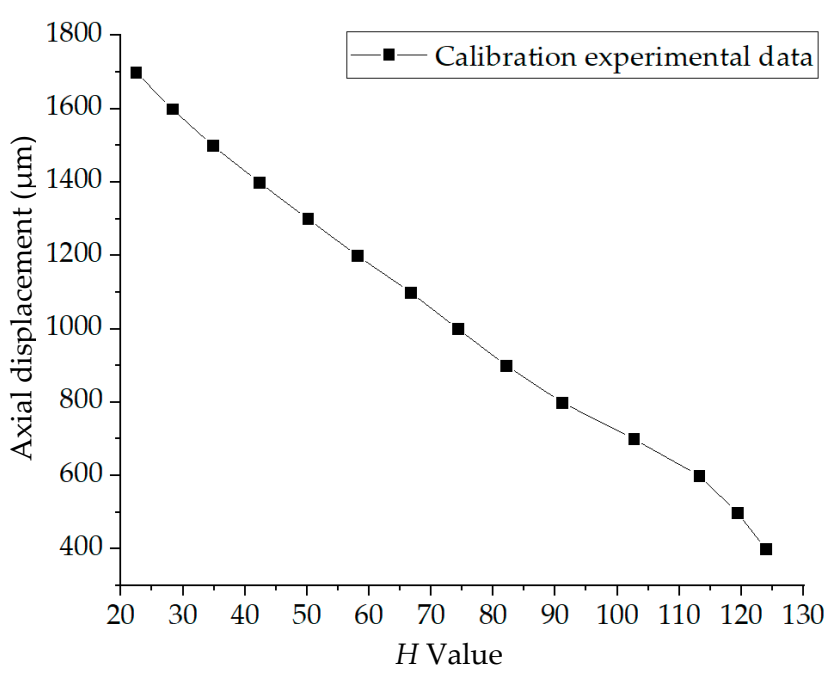

(a)

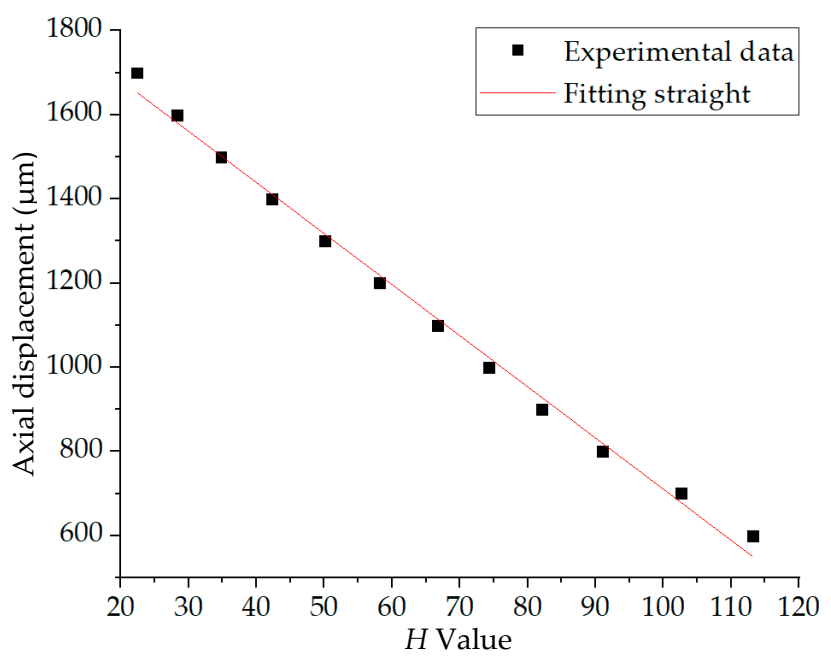

(b)

Figure 11. (a) Calibration experiment result. (b) Linear fitting result.

\subsection{Thickness Measurement for Transparent Specimen}

In Section 2.2.2, the calculation formula for the thickness of the transparent specimen has been proposed. Using the experimental platform shown in Figure 9, the calibration work was performed. Then, two kinds of transparent glass slides with different thicknesses were measured. The physical images of Slide 1 and Slide 2 are shown in Figure 12. The transmission of the two transparent glasses used in this experiment ranged from $84 \%$ (at $380 \mathrm{~nm}$ ) to $90 \%$ (at $717 \mathrm{~nm}$ ).

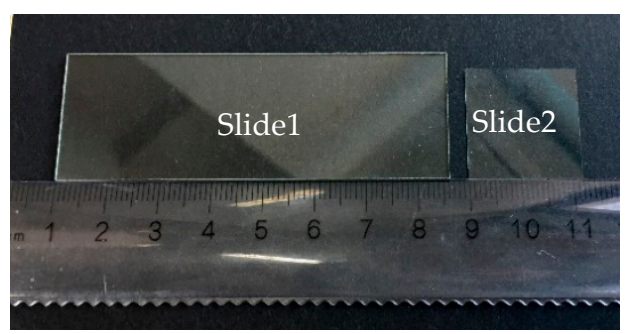

Figure 12. Picture of the transparent glass slides.

\section{(a) Slide 1: thicker transparent specimen}

The true value of Slide 1 was $973.28 \mu \mathrm{m}$, which was measured by the inductance micrometer. In the calibration experiment, two reflected light spots from the upper and lower surface of the slide could be achieved, of which the upper ones were used to calibrate the system. $H_{i}$ and $H_{0}$ of these two light spots were calculated by Equation (16). As the slide moves along the yellow arrow in Figure 9, several groups of $H_{i}$ and $H_{0}$ values can be obtained; however, the differences in each group, which can be represented as $H_{i}-H_{0}$, are not equal. Considering Equation (18), it is known that the thickness of the slide is not only related to the difference between $H_{i}$ and $H_{0}$, but also related to the initial parameters of the measurement system. Therefore, the axial position of the slide along the yellow arrow was fixed. At this axial position, $\theta, a$, and $h_{0}$ were each measured first, and then $\delta$ was calculated through Equation (12), which was 19.1 $1^{\circ}$ The other parameters in Equation (18) are listed in Table 3. 
Table 3. Fixed parameters in Equation (18).

\begin{tabular}{cc}
\hline Parameter & Value \\
\hline$\alpha$ & $58.10^{\circ}$ \\
$\beta$ & $19.90^{\circ}$ \\
$\alpha^{\prime}$ & $34.50^{\circ}$ \\
$\beta^{\prime}$ & $13.10^{\circ}$ \\
$\theta$ & $39.00^{\circ}$ \\
\hline
\end{tabular}

The experiments of the thickness measurement were repeated 40 times; Figure 13 shows one of the 40 captured pictures in the measurement of Slide 1.

Using the color conversion algorithm introduced in Section 2.2.4, the $R, G$, and $B$ information of these 40 pieces of pictures were converted to $H$ values, as listed in Table 4 .

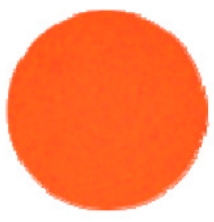

The upper

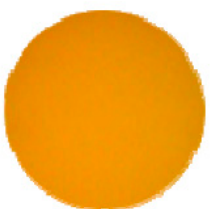

The lower

Figure 13. Experimental picture of Slide 1.

Table 4. Experimental data of the thickness of Slide 1.

\begin{tabular}{cccccccc}
\hline $\begin{array}{c}\text { Piece } \\
\text { Number }\end{array}$ & $\begin{array}{c}\text { H Value } \\
\text { of Upper } \\
\text { Surface }\end{array}$ & $\begin{array}{c}\text { H Value } \\
\text { of Lower } \\
\text { Surface }\end{array}$ & $\begin{array}{c}\text { Value of } \\
\text { Differ- } \\
\text { ence }\end{array}$ & $\begin{array}{c}\text { Piece } \\
\text { Number }\end{array}$ & $\begin{array}{c}\text { H Value } \\
\text { of Upper } \\
\text { Surface }\end{array}$ & $\begin{array}{c}\text { H Value } \\
\text { of Lower } \\
\text { Surface }\end{array}$ & $\begin{array}{c}\text { Value of } \\
\text { Differ- } \\
\text { ence }\end{array}$ \\
\hline 1 & 24.45 & 63.02 & 38.57 & 21 & 24.45 & 63.11 & 38.66 \\
2 & 24.49 & 62.75 & 38.25 & 22 & 24.49 & 63.37 & 38.89 \\
3 & 24.46 & 63.13 & 38.67 & 23 & 24.06 & 63.10 & 39.04 \\
4 & 24.49 & 63.10 & 38.60 & 24 & 24.49 & 63.14 & 38.64 \\
5 & 24.47 & 63.13 & 38.66 & 25 & 24.07 & 63.16 & 39.09 \\
6 & 24.50 & 63.01 & 38.52 & 26 & 24.45 & 63.11 & 38.66 \\
7 & 24.46 & 63.11 & 38.66 & 27 & 24.49 & 63.06 & 38.57 \\
8 & 24.49 & 63.12 & 38.63 & 28 & 24.45 & 63.08 & 38.63 \\
9 & 24.48 & 63.08 & 38.59 & 29 & 24.49 & 63.11 & 38.62 \\
10 & 24.46 & 63.16 & 38.70 & 30 & 24.46 & 63.10 & 38.63 \\
11 & 24.49 & 62.94 & 38.45 & 31 & 24.49 & 63.05 & 38.56 \\
12 & 24.49 & 63.17 & 38.69 & 32 & 24.49 & 63.40 & 38.91 \\
13 & 24.46 & 63.01 & 38.55 & 33 & 24.45 & 63.12 & 38.67 \\
14 & 24.48 & 63.13 & 38.65 & 34 & 24.48 & 63.13 & 38.65 \\
15 & 24.49 & 63.01 & 38.51 & 35 & 24.49 & 63.37 & 38.87 \\
16 & 24.45 & 63.18 & 38.73 & 36 & 24.50 & 63.07 & 38.57 \\
17 & 24.49 & 62.96 & 38.47 & 37 & 24.45 & 63.09 & 38.64 \\
18 & 24.07 & 63.15 & 39.09 & 38 & 24.49 & 63.12 & 38.63 \\
19 & 24.49 & 62.96 & 38.47 & 39 & 24.48 & 63.09 & 38.61 \\
20 & 24.07 & 63.20 & 39.12 & 40 & 24.46 & 63.14 & 38.68 \\
\hline The average value of & & 38.67 & & Relative & & & \\
the differor $(\sigma)$ & & & 0.18 \\
\hline
\end{tabular}

Based on the discussion in Sections 2.2.3 and 3.1.1, $\theta$ was determined as $39^{\circ}$, and the value of $k_{1}$ is 12.14 expressed in Equation (18). Calculated with the data in Tables 4 and 5 with Equation (18), the thickness of Slide 1 was $998.82 \mu \mathrm{m}$. The calculation formula of $\sigma$ is as follows:

$$
\sigma=\sqrt{\frac{\sum\left(x_{i}-\bar{x}\right)^{2}}{n-1}}
$$


The repeatability was $0.54 \mu \mathrm{m}$, measured by $3 \sigma$, and the measurement error was $2.62 \%$.

(b) Slide 2: thinner transparent specimen

Based on the measurement of the thicker slide in part (a), the measurement of the 170-190 $\mu \mathrm{m}$ slide was carried out. The experiments of the thickness measurement were also repeated 40 times; Figure 14 shows one of the 40 captured pictures in the measurement of Slide 2.

The true value of Slide 2 is $181.08 \mu \mathrm{m}$, measured by the inductance micrometer. The results were measured by the self-built instrument and are listed in Table 5 .

The data in Table 5 were processed in the same way as for Slide 1 , and the thickness was calculated to be $187.00 \mu \mathrm{m}$. The repeatability was $0.75 \mu \mathrm{m}$, measured by $3 \sigma$, and the measurement error was $3.27 \%$.

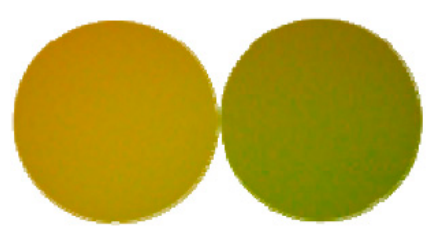

The upper The lower

Figure 14. Experimental picture of Slide 2.

Table 5. Experimental data of the thickness of Slide 2.

\begin{tabular}{cccccccc}
\hline $\begin{array}{c}\text { Piece } \\
\text { Number }\end{array}$ & $\begin{array}{c}\text { H Value } \\
\text { of Upper } \\
\text { Surface }\end{array}$ & $\begin{array}{c}\text { H Value } \\
\text { of Lower } \\
\text { Surface }\end{array}$ & $\begin{array}{c}\text { Value of } \\
\text { Differ- } \\
\text { ence }\end{array}$ & $\begin{array}{c}\text { Piece } \\
\text { Number }\end{array}$ & $\begin{array}{c}\text { H Value } \\
\text { of Upper } \\
\text { Surface }\end{array}$ & $\begin{array}{c}\boldsymbol{H} \text { Value } \\
\text { of Lower } \\
\text { Surface }\end{array}$ & $\begin{array}{c}\text { Value of } \\
\text { Differ- } \\
\text { ence }\end{array}$ \\
\hline 1 & 52.07 & 59.49 & 7.42 & 21 & 52.03 & 59.48 & 7.45 \\
2 & 52.02 & 58.91 & 6.89 & 22 & 52.08 & 59.44 & 7.36 \\
3 & 52.09 & 59.51 & 7.42 & 23 & 52.03 & 59.36 & 7.32 \\
4 & 52.05 & 58.90 & 6.85 & 24 & 52.09 & 59.45 & 7.35 \\
5 & 52.05 & 59.40 & 7.35 & 25 & 52.12 & 59.40 & 7.29 \\
6 & 52.06 & 58.57 & 6.51 & 26 & 52.09 & 59.40 & 7.32 \\
7 & 52.08 & 59.51 & 7.43 & 27 & 52.04 & 59.48 & 7.44 \\
8 & 52.04 & 58.88 & 6.84 & 28 & 52.06 & 59.50 & 7.43 \\
9 & 52.06 & 59.44 & 7.38 & 29 & 52.05 & 58.98 & 6.93 \\
10 & 52.06 & 59.40 & 7.34 & 30 & 52.07 & 59.52 & 7.45 \\
11 & 52.07 & 59.47 & 7.41 & 31 & 52.06 & 59.44 & 7.37 \\
12 & 52.08 & 58.95 & 6.87 & 32 & 52.07 & 59.48 & 7.41 \\
13 & 52.05 & 59.50 & 7.44 & 33 & 52.10 & 59.45 & 7.34 \\
14 & 52.01 & 58.94 & 6.93 & 34 & 52.07 & 59.45 & 7.38 \\
15 & 52.07 & 59.41 & 7.34 & 35 & 52.05 & 58.91 & 6.86 \\
16 & 52.07 & 58.90 & 6.83 & 36 & 52.12 & 59.49 & 7.37 \\
17 & 52.09 & 59.46 & 7.37 & 37 & 52.08 & 59.43 & 7.35 \\
18 & 52.10 & 59.47 & 7.37 & 38 & 52.06 & 59.46 & 7.40 \\
19 & 52.07 & 59.45 & 7.37 & 39 & 52.04 & 58.95 & 6.91 \\
20 & 52.04 & 58.96 & 6.91 & 40 & 52.09 & 59.50 & 7.41 \\
\hline The average value of & & 7.24 & & Relative & & & \\
the differor $(\sigma)$ & & 0.25 & \\
\hline
\end{tabular}

\section{Discussions}

The experimental results showed that the axial measurement accuracy of the system could reach the micron level and the repeatability is better than $0.75 \mu \mathrm{m}$. However, there is a high requirement for the position of all the optical parts in this system, and we will try to improve the adaptability of the system in future works.

The relationship between the thickness $d$ and the angle $\theta$ is discussed and derived in this paper. We calculate the value of $\theta$ through the values of $d_{\min }$ and $d_{\max }$, which is $39^{\circ}$. 
The transmission of the two transparent glass slides used in this experiment ranged from $84 \%$ to $90 \%$. The current experiments have requirements for the transmission, flatness of transparent specimens, and the stability of the measuring system. In the future, related studies and quantificational analyses will be conducted for different materials.

The application scope of this paper is mainly the measurement of transparent specimens. In the future, not only the relationship of inclined angle $\theta$ and thickness measurement range, but also the influence of attenuation range and refractive index of transparent specimens in the system, will be further discussed.

\section{Conclusions}

In this paper, a novel chromatic confocal system with inclined illumination is proposed. The mathematic model of the thickness measurement for the transparent specimens was built, and the influence of the inclined angle $\theta$ was discussed. The experimental results show that the axial measurement accuracy reached the micron level and the repeatability was better than $0.75 \mu \mathrm{m}$.

Author Contributions: Conceptualization, Q.Y. and Y.Z.; methodology, Q.Y.; software, C.W.; validation, Y.Z., W.S. and S.D.; formal analysis, W.S.; investigation, Y.W.; resources, S.D.; data curation, F.C.; writing-original draft preparation, Y.Z.; writing-review and editing, T.L. All authors have read and agreed to the published version of the manuscript.

Funding: This study was supported by the National Natural Science Foundation of China (no. 52075190 and no. 62075067); the Science and Technology Program of Fujian, China (no. 2019I0013); and the Promotion Program for Young and Middle-Aged Teachers in Science and Technology Research of Huaqiao University (no. ZQN-PY604).

Institutional Review Board Statement: Not applicable.

Informed Consent Statement: Not applicable.

Data Availability Statement: Data sharing is not applicable to this article.

Conflicts of Interest: The authors declare no conflict of interest.

\section{References}

1. Wang, Y.; Xi, M.; Liu, H.; Ding, Z.; Du, W.; Meng, X.; Sui, Y.; Li, J.; Jia, Z. On-machine noncontact scanning of high-gradient freeform surface using chromatic confocal probe on diamond turning machine. Opt. Laser Technol. 2021, 134, 106569. [CrossRef]

2. Hillenbrand, M.; Lorenz, L.; Kleindienst, R.; Grewe, A.; Sinzinger, S. Spectrally multiplexed chromatic confocal multipoint sensing. Opt. Lett. 2013, 38, 4694-4697. [CrossRef]

3. Chun, B.; Kim, K.; Gweon, D. Three-dimensional surface profile measurement using a beam scanning chromatic confocal microscope. Rev. Sci. Instrum. 2009, 80, 073706. [CrossRef]

4. Du, H.; Zhang, W.; Ju, B.; Sun, Z.; Sun, A. A new method for detecting surface defects on curved reflective optics using normalized reflectivity. Rev. Sci. Instrum. 2020, 91, 036103. [CrossRef]

5. Zou, J.; Yu, Q.; Cheng, F. Differential chromatic confocal roughness evaluation system and experimental research. Chin. Opt. 2020, 13, 1103-1114.

6. Fu, S.; Kor, W.S.; Cheng, F.; Seah, L.K. In-situ measurement of surface roughness using chromatic confocal sensor. Procedia CIRP 2020, 94, 780-784. [CrossRef]

7. Berkovic, G.; Zilberman, S.; Shafir, E.; Rubin, D. Chromatic confocal displacement sensing at oblique incidence angles. Appl. Opt. 2020, 59, 3183-3186. [CrossRef] [PubMed]

8. Bai, J.; Li, X.; Wang, X.; Zhou, Q.; Ni, K. Chromatic Confocal Displacement Sensor with Optimized Dispersion Probe and Modified Centroid Peak Extraction Algorithm. Sensors 2019, 19, 3592. [CrossRef] [PubMed]

9. Chen, C.; Wang, J.; Liu, X.; Lu, W.; Zhu, H.; Jiang, X. Influence of sample surface height for evaluation of peak extraction algorithms in confocal microscopy. Appl. Opt. 2018, 57, 6516-6526. [CrossRef] [PubMed]

10. Sun, H.; Wang, S.; Bai, J.; Zhang, J.; Huang, J.; Zhou, X.; Liu, D.; Liu, C. Confocal laser scanning and 3D reconstruction methods for the subsurface damage of polished optics. Opt. Lasers Eng. 2021, 136, 106315. [CrossRef]

11. Hillenbrand, M.; Weiss, R.; Endrody, C.; Grewe, A.; Hoffmann, M.; Sinzinger, S. Chromatic confocal matrix sensor with actuated pinhole arrays. Appl. Opt. 2015, 54, 4927-4936. [CrossRef] [PubMed]

12. Luo, D.; Taphanel, M.; Claus, D.; Boettcher, T.; Osten, W.; Längle, T.; Beyerer, J. Area scanning method for 3D surface profilometry based on an adaptive confocal microscope. Opt. Lasers Eng. 2020, 124, 105819. [CrossRef] 
13. Li, S.; Liang, R. DMD-based three-dimensional chromatic confocal microscopy. Appl. Opt. 2020, 59, 4349-4356. [CrossRef] [PubMed]

14. Hillenbrand, M.; Mitschunas, B.; Brill, F.; Grewe, A.; Sinzinger, S. Spectral characteristics of chromatic confocal imaging systems. Appl. Opt. 2014, 53, 7634-7642. [CrossRef] [PubMed]

15. Lu, W.; Chen, C.; Wang, J.; Richard, L.; Zhang, C. Characterization of the displacement response in chromatic confocal microscopy with a hybrid radial basis function network. Opt. Express 2019, 27, 22737-22752. [CrossRef] [PubMed]

16. Bai, J.; Li, X.; Wang, X.; Wang, J.; Ni, K.; Zhou, Q. Self-reference dispersion correction for chromatic confocal displacement measurement. Opt. Lasers Eng. 2021, 140, 106540. [CrossRef]

17. Miks, A.; Novak, J.; Novak, P. Analysis of method for measuring thickness of plane-parallel plates and lenses using chromatic confocal sensor. Appl. Opt. 2010, 49, 3259-3264. [CrossRef]

18. Boettcher, T.; Gronle, M.; Osten, W. Single-shot multilayer measurement by chromatic confocal coherence tomography. Opt. Metrol. 2017, 10329, 103290K.

19. Yu, Q.; Zhang, K.; Cui, C.; Zhou, R.; Cheng, F.; Ye, R.; Zhang, Y. Method of thickness measurement for transparent specimens with chromatic confocal microscopy. Appl. Opt. 2018, 57, 9722-9728. [CrossRef]

20. Li, J.; Zhao, Y.; Du, H.; Zhu, X.; Wang, K.; Zhao, M. Adaptive modal decomposition based overlapping-peaks extraction for thickness measurement in chromatic confocal microscopy. Opt. Express 2020, 28, 36176-36187. [CrossRef]

21. Taphanel, M.; Beyerer, J. Fast 3D In-line Sensor for Specular and Diffuse Surfaces Combining the Chromatic Confocal and Triangulation Principle. In Proceedings of the Conference Record IEEE Instrumentation \& Measurement Technology Conference, Graz, Austria, 13-16 May 2012.

22. Taphanel, M.; Zink, R.; Längle, T.; Beyerer, J. Multiplex acquisition approach for high speed 3D measurements with a chromatic confocal microscope. SPIE Opt. Metrol. 2015, 9525, 95250Y.

23. Seung, W.L.; Garam, C.; Sin, Y.L.; Yeongchan, C.; Heui, J.P. Coaxial spectroscopic imaging ellipsometry for volumetric thickness measurement. Appl. Opt. 2021, 60, 67-74. 